\title{
An error function minimization approach for the inverse problem of adaptive mirrors tuning.
}

\author{
Maurizio Vannoni*a ${ }^{\mathrm{a}}$, Fan Yang ${ }^{\mathrm{a}}$, Frank Siewert ${ }^{\mathrm{b}}$, Harald Sinn ${ }^{\mathrm{a}}$ \\ ${ }^{a}$ European XFEL GmbH, Albert-Einstein-Ring 19, 22761 Hamburg, Germany; \\ ${ }^{b}$ Helmholtz Zentrum Berlin, BESSY-II, Institute for Nanometre Optics and Technology, Albert- \\ Einstein-Str. 15, 12489 Berlin, Germany
}

\begin{abstract}
Adaptive X-ray optics are more and more used in synchrotron beamlines, and it is probable that they will be considered for the future high-power free-electron laser sources, as the European XFEL now under construction in Hamburg, or similar projects now in discussion. These facilities will deliver a high power x-ray beam, with an expected high heat load delivered on the optics. For this reason, bendable mirrors are required to actively compensate the resulting wavefront distortion. On top of that, the mirror could have also intrinsic surface defects, as polishing errors or mounting stresses. In order to be able to correct the mirror surface with a high precision to maintain its challenging requirements, the mirror surface is usually characterized with a high accuracy metrology to calculate the actuators pulse functions and to assess its initial shape. After that, singular value decomposition (SVD) is used to find the signals to be applied into the actuators, to reach the desired surface deformation or correction. But in some cases this approach could be not robust enough for the needed performance. We present here a comparison between the classical SVD method and an error function minimization based on root-mean-square calculation. Some examples are provided, using a simulation of the European XFEL mirrors design as a case of study, and performances of the algorithms are evaluated in order to reach the ultimate quality in different scenarios. The approach could be easily generalized to other situations as well.
\end{abstract}

Keywords: $\mathrm{x}$-rays, adaptive mirror, metrology, error function, SVD

\section{INTRODUCTION}

Because of the high demanding requirements asked nowadays for modern x-rays sources, it is really challenging to manufacture mirrors that are complying these specifications without any need of adjustment or tuning. Also, in some special applications, there is a need of dynamic adjustments of the optics to allow compensation of other effects. For example, using the source at different intensities and energies means that the beam conditions will change during the operations and some effects that cannot be completely known and considered in the preliminary design stage will probably appear. One good example is the future European XFEL source, a powerful free-electron laser currently on construction in Hamburg. Such a beam will have some peculiarities, as the high transversal coherence, repetition rate and power level: these properties will be used to support new and interesting experiments, as high-speed diffraction experiments to study physical and chemical processes in the time-domain, or creation and study of extreme states of the matter that can be reached with these energy levels. The beam will be transported and shared among the different beam paths (generically called "beamlines") through a distribution mirror system (Fig. 1).

Every different beamline, after the undulator section, will start with a couple of mirrors used in grazing incidence. The first mirror will adsorb the high harmonics radiation, reflecting the energy that we are interested in. The second mirror, together with the first, will point the beam towards the experiment station. These mirrors will adsorb part of the beam, together with the residual high-harmonic radiation, and this partial adsorption along the surfaces will produce a thermal bump, causing a non-perfect flat surface and an incorrect reflection. In the case of a free-electron laser, surface modifications and bending will be immediately visible along the beam and, in the worst case, they will reduce the coherence properties of the beam, resulting also in an incorrect focus. The amount of thermal bump will change, depending on the beam energy and the grazing incidence angle, so it is not possible to adjust the situation for a single operating mode. For this reason, every second mirrors will be bendable and deformed in a controlled way, in order to correct the deformations introduced by the thermal bump. These mirrors are generally named "bendable mirrors" or "adaptive mirrors" and they can be produced in few different ways. However, the basic principle to build such mirrors is 
to introduce some benders, in different points of the surface, to bend locally the mirror in order to produce the needed correction. Such a correction is different, depending on the operational mode, characteristics of the beam, quantity of power, and energy. Having the possibility to change locally the surface could be even more effective to recover other error sources. For example, we could correct positioning errors, residual stresses, and mechanical drifts of the mirrors that could arise along the time.

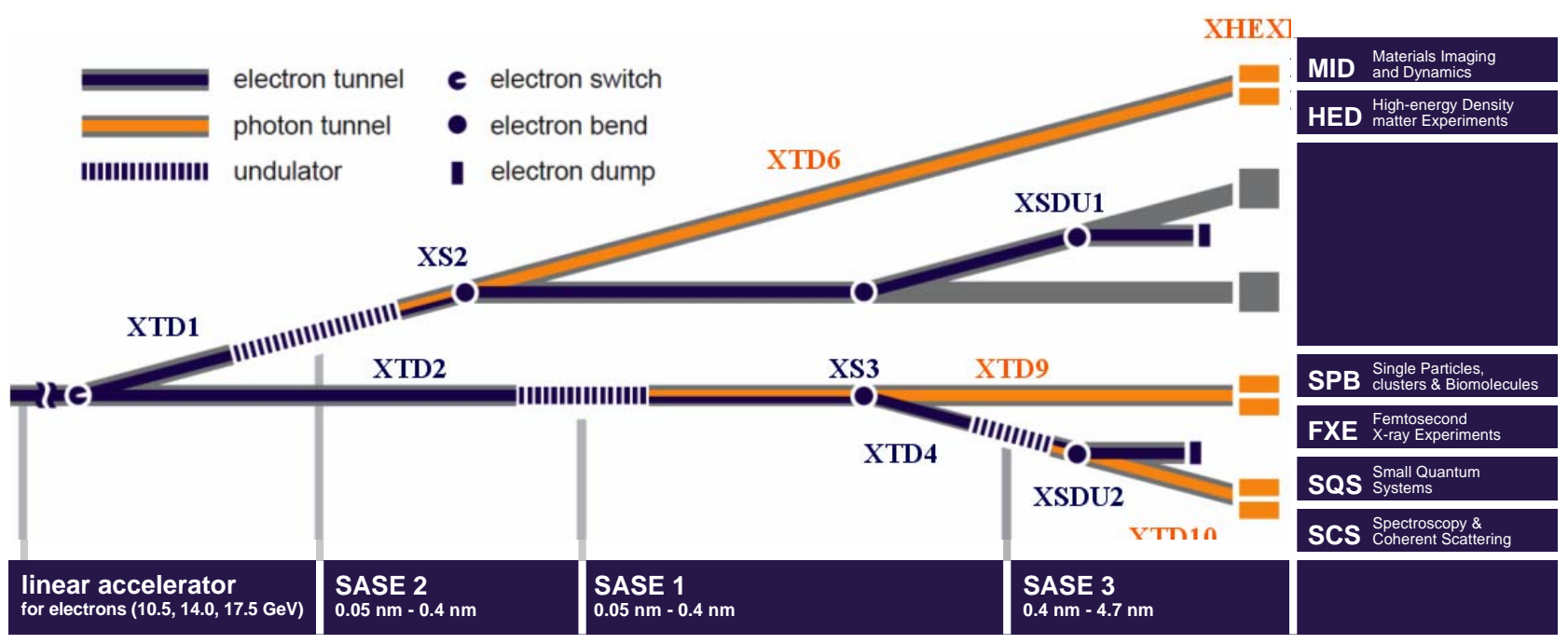

Figure 1. General scheme of European XFEL project beamlines.

The importance of such bendable mirrors is important even in other fields: as an example, for astronomical big telescopes. Such mirrors are there widely used in the so called "active mirrors", to compensate the mechanical stresses of the main mirror when used with different azimuthal angles respect to the gravity, but also in the so called "adaptive mirrors", to compensate the atmospheric drift and refractive index changes. The two mirror concepts are similar, both have actuators as controllable local adjusters, but they need different characteristics. Active mirrors are with a higher range of correction, but slowly controlled and open loop, while the adaptive mirrors are faster and mainly used in a closed loop with wavefront sensors, to correct the rapid atmospheric changes.

In our case, for synchrotron and free electron lasers applications, we are a bit in between. The required adjustments are very slow, we do not have to compensate a fast changing effect, but the precision required is high and we want to have the maximum performance theoretically possible. Because of the application, we need a sub-nanometer resolution, and mainly distributed on the length axis. In case of x-rays optics, the grazing incidence configuration means that the beam is illuminating the mirror on a very long stripe: one axis is longer than the other one, by a factor of two or even three order of magnitudes. For this reason the adjustments are only placed along that direction. Also, the needed adjustments can be made with a slow time rate, but we need to adjust the situation to have the best mirror correction that we can do with the provided benders. To accomplish that, a mathematical approach is generally used. We present here a comparison between two different algorithms: the classical matrix single-value-decomposition (SVD) approach and an error-function minimization strategy. We will explain the differences between the two approaches, explaining the strengths and weaknesses of both and provide several examples of corrections, to evaluate the performance of both algorithms.

\section{BENDABLE MIRRORS DISCRETE INVERSE PROBLEM AND SVD APPROACH}

Generally speaking, the inverse problem of adaptable mirrors tuning is classically solved with single value decomposition method (SVD). Let's take a mirror with $M$ actuators, placed along its length. The actuators are designed in a way that they can generate a controllable local bending, applying for example an electrical signal. Such benders are usually built using piezoelectric devices, in an open loop system. The characterization of the bendable mirror is done in the lab, using some specific instruments as pentaprism profilometers, or directly inside the beamline using the beam as a 
measurement tool, for example with the ray-pencil method. In both cases the approach is conceptually similar. First, we measure the mirror profile without any correction, and we store such profile as a reference baseline. After that, we move the first bender applying a fixed signal: in case of a piezoelectric bender we apply a certain high-voltage signal. We measure the resulting profile, and we calculate the difference between this profile and the stored baseline. The result is named "pulse profile" and can be measured for the j-nth bender, resulting in profile $A_{j}$. If $N$ is the number of points measured in every pulse function and in the baseline profile, $A_{j} \in \square^{N}$. We assume here that the pulse profile and the high-voltage signal are in a completely full linear regime. Under this assumption, we can divide every pulse function for the voltages applied, obtaining a "unit impulse" result, and we can create a matrix, classically named "interaction matrix", putting together the scaled $M$ pulse profiles evaluated in $N$ points, having at the end $A \in \square^{N \times M}$. Now, if we have a given profile $b \in \square^{N}$, we can solve the linear system $A V=-b$ to find the needed voltages vector $V \in \square^{M}$ to correct $b$ in order to have at the end a flat profile. Again, the linearity of the problem is implicitly assumed. Because $A \in \square^{N \times M}$, it is in general not a square matrix, and we cannot simply compute its inverse. But the solution can be found using singular value decomposition:

$$
\begin{gathered}
A v-b=0 \\
A^{T} A v=-A^{T} b \\
\left(A^{T} A\right)^{-1}\left(A^{T} A\right) v=-\left(A^{T} A\right)^{-1} A^{T} b \\
v=-\left(A^{T} A\right)^{-1} A^{T} b
\end{gathered}
$$

This method has been widely used with bendable mirrors, because it is fast and easy to implement. However, there are two major problems to consider.

First, the bendable mirrors, especially the ones designed with mechanical or piezo benders, have some mechanical limitations to be considered while they are used. Usually, there is a maximum amount of signal, resulting in a maximum amount of correction that can be applied. Also, in case of mechanical or piezo benders, there is a limit in the amount of local amount of stress that can be supported by the mirror itself. Speaking about high-voltage signals in case of a piezo driven mirror, it means that we have to put a maximum amount of signal allowed, in both directions, and a maximum difference of signal between two adjacent benders. For the majority of piezo driven bendable mirror, it means to consider a maximum signal of 2000 volt, and a maximum difference between two adjacent piezos of 500 volt. These constraints are simply not considered by SVD mathematical approach, and this originates often a number of not acceptable results.

Second problem, the linear system in Eq. 1 is not fully correct in most of the grazing incidence x-ray mirrors. Infact, the mechanical mounting for such mirrors has usually a rotation control, to allow a correct aiming of the beam. Also, a rigid translation stage in the direction normal to the mirror surface is needed, also to allow intercepting correctly the incidence beam. These two degrees of freedom, rotation and translation, are mathematically equivalent to a best plane removal of the mirror surface. It means that the correction that we want to apply to the mirror surface, designed to obtain a perfect flat, can also produce a perfect plane with a generic orientation. Mathematically, the Eq. 1 should be re written as

$$
A v-b=\left(a_{0} x+b_{0}\right)
$$

with $X$ the coordinate vector and $a_{0}, b_{0}$ scalar values. The resulting system is ill-conditioned from a linear system point of view: in other words, it has no unique solution anymore. Therefore, instead of computing a unique solution of the problem, we have to switch to "searching for the better solution" in a least squares fitting meaning.

\section{ERROR FUNCTION MINIMIZATION APPROACH}

To solve the issues related to SVD approach, we propose a different method. We build an error function, to be evaluated on the profile that we have, calculated as the root-mean-square of the difference between that profile and the profile that we want to obtain (in our case, a perfectly flat surface). The best plane is fitted and removed, before calculating the rms error. In the function, we also consider the different constraints that we have, in terms of maximum amount of signal allowed and maximum amount of difference between adjacent benders signals. In case one of the constraints is violated, 
we assign an infinite value on the error function. We putted such an error function inside a general minimization algorithm, as Nelder-Mead simplex algorithm, Powell or Monte-Carlo. We start the minimization from a set of values that are inside the given constraints, in general even a zero set could be used, and we start the minimization algorithm. The algorithm runs for a certain number of iterations, depending on the precision that we need, and at the end we obtain a vector of voltage corrections that we can use to calculate the resulting profile. The schematic of the error function that has to be minimized is in Fig. 2.

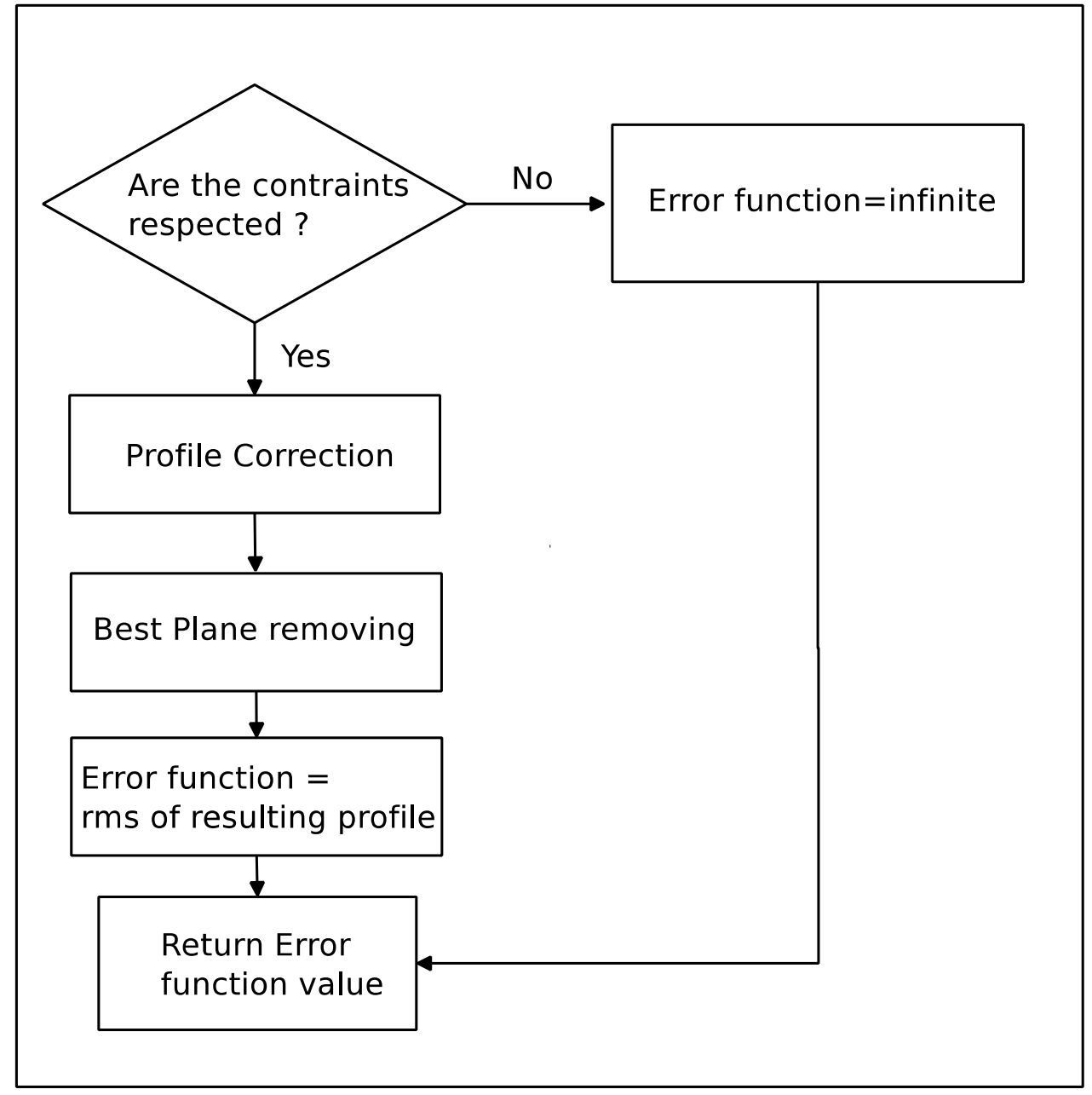

Figure 2. Error function definition.

The total time needed to reach a minimum for the algorithm is ranging from few minutes to half an hour in the worst case that we have tried, on a standard office notebook (1.7 Ghz speed processor, 4Gb RAM memory).

\section{METHODS COMPARISON}

To show the peculiarities of the two methods, we can check the results on some example profiles. We have used here the pulse functions calculated with Finite Element Analysis in the case of a distribution mirror for European XFEL project. The mirror is almost $900 \mathrm{~mm}$ long, and we have 18 piezo elements placed to control the bending. The first two and the last two piezo are connected together, so we have a total of 16 voltage channels that we can optimize. After having calculated the interaction matrix, we apply the two correction methods to a gaussian profile, to simulate a possible gaussian bending due to beam partial adsorption, and an astigmatic profile, to simulate a mounting stress. The gaussian profile is calculated ad hoc, while the astigmatic profile is based on some preliminary measurements currently on going on a real prototype of the mirror. 


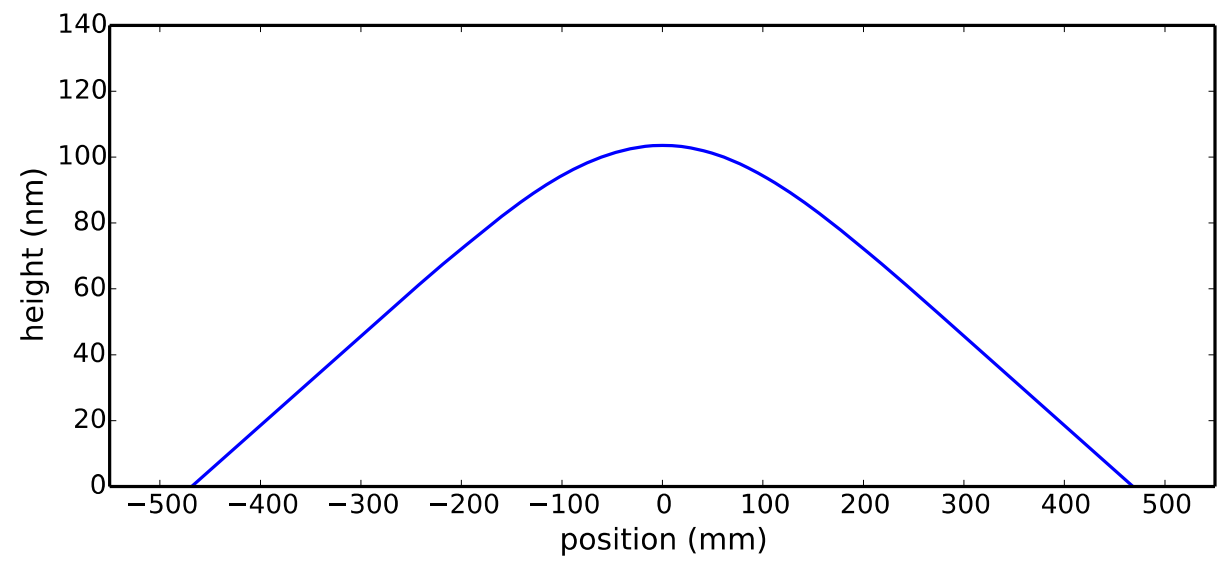

Figure 3. Gaussian profile to be corrected.
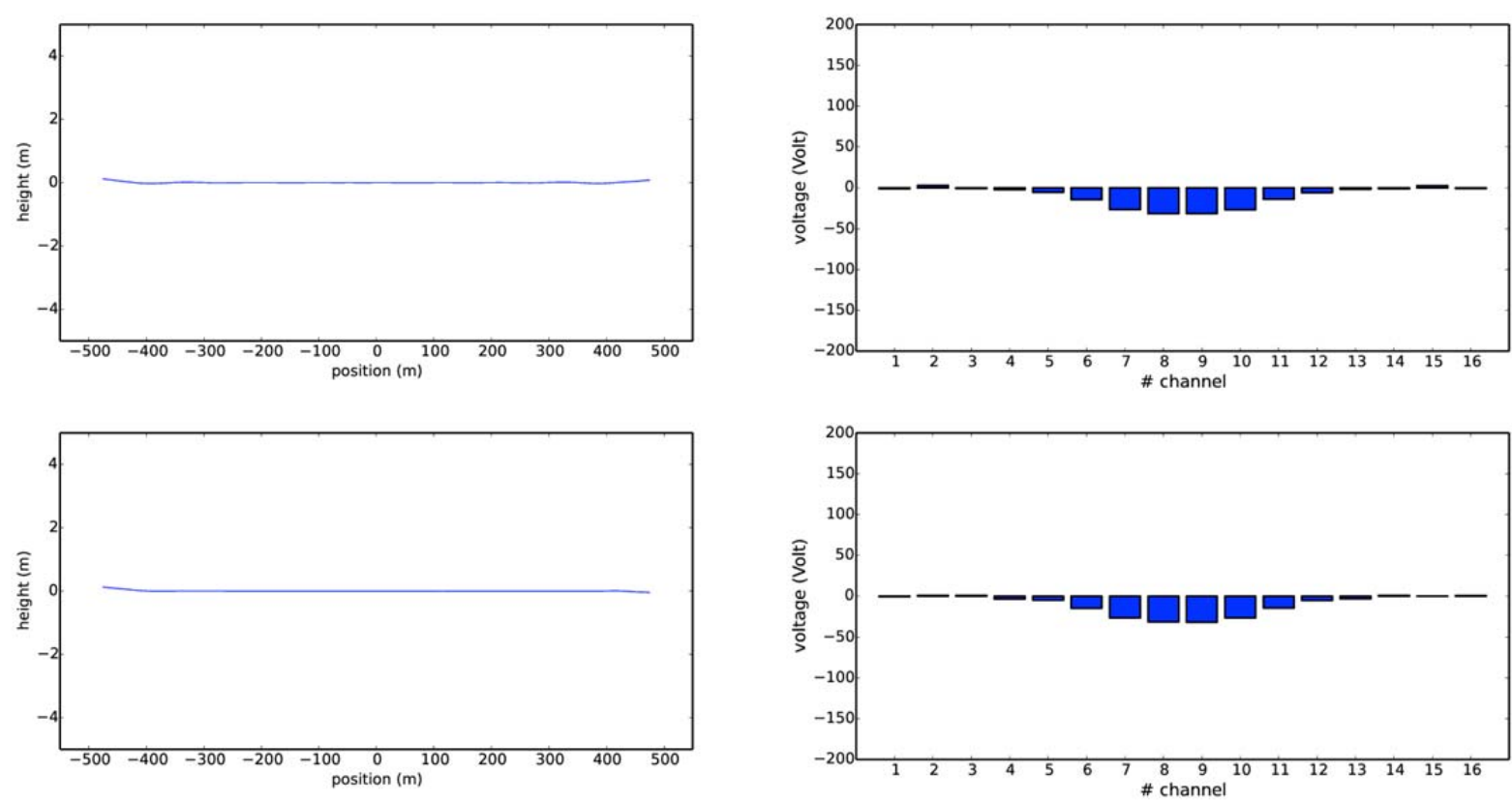

Figure 4. Corrections results. Top-left: result with SVD correction. Top-right: voltage values (in red the ones that are not allowed due to the constraints. Bottom-left: result with error function correction. Bottom-right: voltage values. 


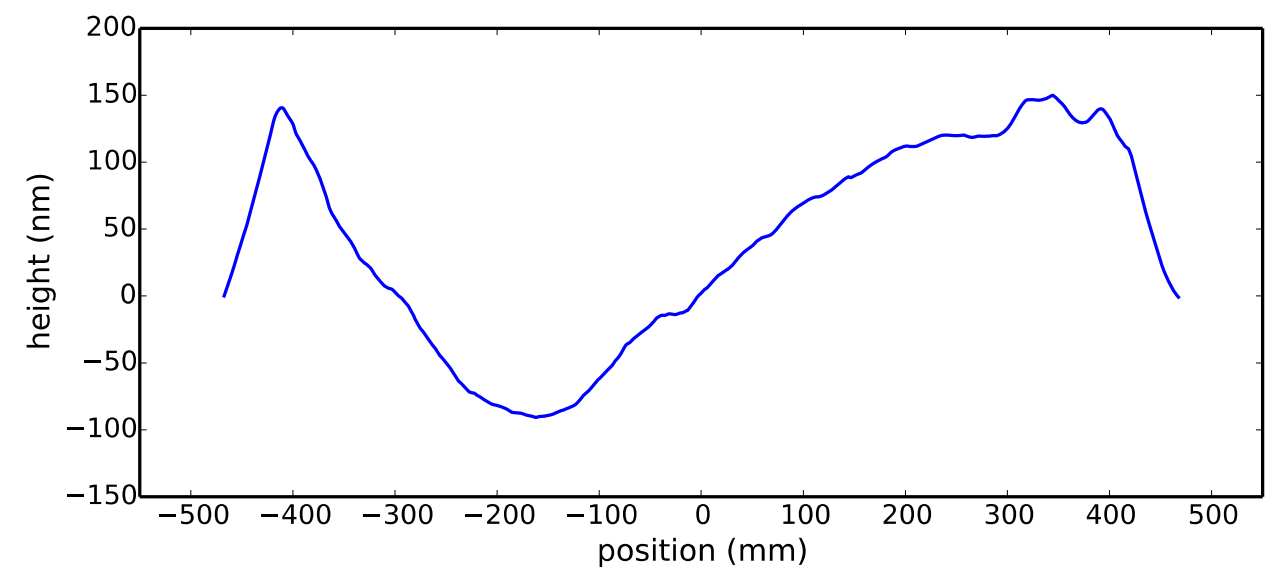

Figure 5. Astigmatic profile to be corrected.
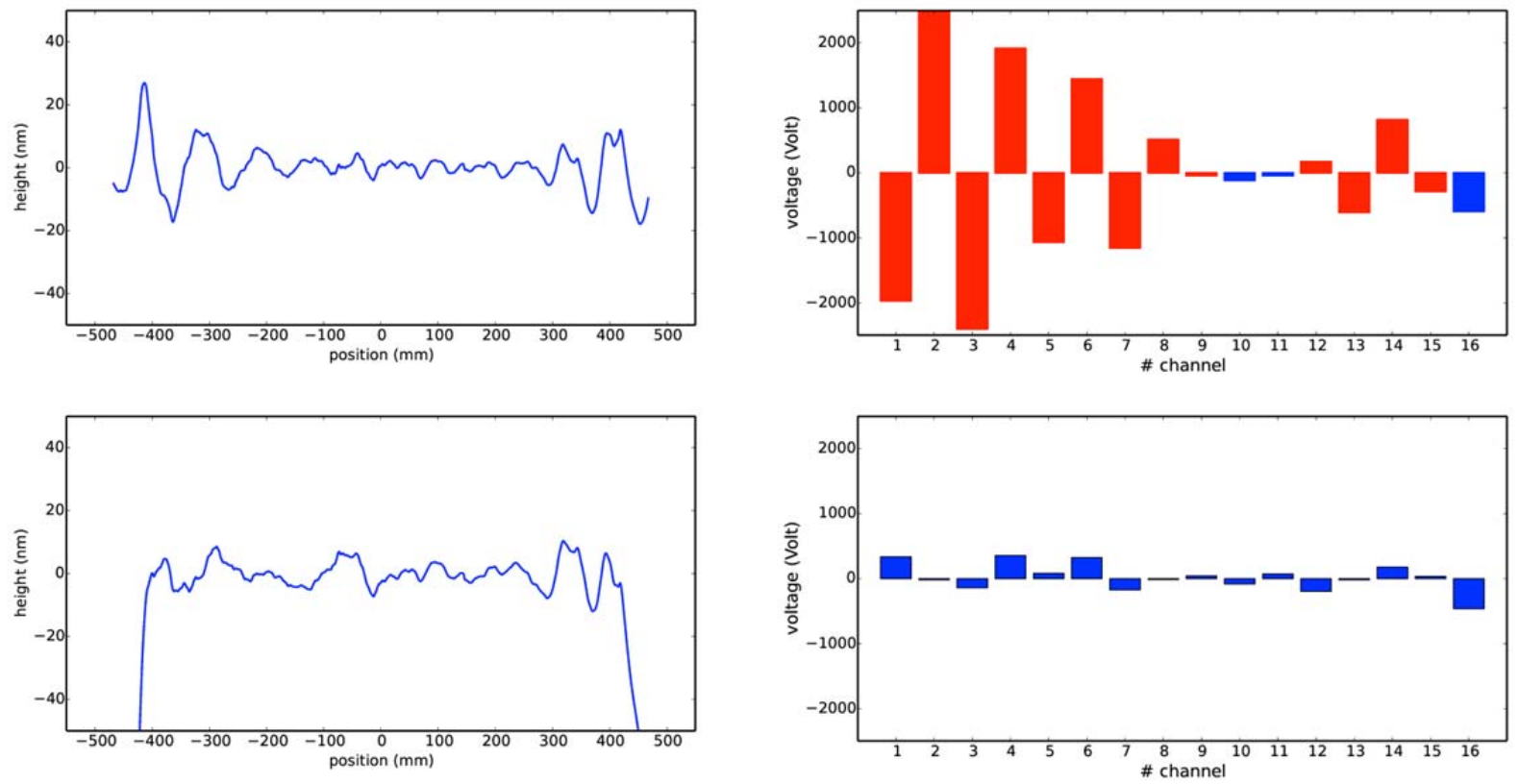

Figure 6. Corrections results. Top-left: result with SVD correction. Top-right: voltage values (in red the ones that are not allowed due to the constraints. Bottom-left: result with error function correction. Bottom-right: voltage values.

As we can easily see, both the algorithms can easily correct the Gaussian profile, while the astigmatic profile is corrected with a feasible solution only by the error function method. The SVD is still finding a solution, but this solution is not applicable due to the physical constraints.

\section{CONCLUSIONS}

A method based on an error function minimization to simulate bimorph and in general bendable mirrors is reported. Using a proper model of pulse functions, obtained with FEA analysis, we built a proper error function to be minimized. An example mirror behavior has been then simulated, and a particular minimization strategy is used to forecast the amount of correction that we could have in some particular cases. The cases that we examined are related to XFEL project, a Free Electron Laser being built in Hamburg. We showed that this algorithm could overcome easily the classical SVD approach, in particular when the amount of correction to do is high and not uniform. The algorithm is built in a way 
that could be easily upgraded with real pulse functions, measured with long trace profilers or interferometers. In our present study, this method will be very useful to evaluate future XFEL bendable mirrors performance, in order to be able to correct heating effects caused by the x-ray beam and possible polishing and mechanical misalignment effects.

\section{REFERENCES}

[1] Susini, J., Labergerie D., Zhang L., "Compact active/adaptive xray mirror: Bimorph piezoelectric flexible mirror," Review of Scientific Instruments 66, 2229-2231 (1995).

[2] R. Signorato, "R\&D program on multisegmented piezoelectric bimorph mirrors at the ESRF: status report," Proc. SPIE 3447, Advances in Mirror Technology for Synchrotron X-Ray and Laser Applications, 20 (1998).

[3] R. Signorato, J.-F. Carre, and T. Ishikawa, "Performance of the SPring-8 modular piezoelectric bimorph mirror prototype", Proc. SPIE 4501, 76 (2001).

[4] R. Signorato, D. Hausermann, M. Somayazulu and J.-F. Carre, "Performance of an adaptive u-focusing Kirkpatrick-Baez system for high-pressure studies at the Advanced Photon Source", Proc. SPIE 5193, 112 (2004).

[5] U. Hahn and H. Schulte-Schrepping, "Mirror benders for white beam collimation at HASYLAB", Proc. SPIE 3447, 12 (1998).

[6] H. Padmore, M. R. Howells, S. Irick, T. Renner, R. Sandler, and Y. M. Koo, "Some new schemes for producing high-accuracy elliptical X-ray mirrors by elastic bending", Proc. SPIE 2856, Optics for High-Brightness Synchrotron Radiation Beamlines II, 145 (1996).

[7] R. Signorato, O. Hignette, J. Goulon "Multi-segmented piezoelectric mirrors as active/adaptive optics components", Journal of Synchrotron radiation 5, 797-800, (1998).

[8] S. Yuan, K. Goldberg, V. Yashchuk, R. Celestre, T. Warwick, W. McKinney, G. Morrison, S. Rekawa, I. Mochi, and H. Padmore, "At-Wavelength and Optical Metrology of Bendable X-Ray Optics for Nanofocusing at the ALS," in Frontiers in Optics 2009/Laser Science XXV/Fall 2009 OSA Optics \& Photonics Technical Digest, OSA Technical Digest (CD) (Optical Society of America, 2009), paper FThT2.

[9] Societe Europenne de Systemes Optiques S.E.S.O, Patent EP1818711 (B1), "Bimorph mirror comprising two piezoelectric layers" (2009).

[10]F. Yang, H. Sinn, A. Trapp, R. Signorato, and T. Noll, "FEA analysis of the adaptive mirror design for the European XFEL beam transport," MEDSI 2012, Shanghai, China (2012).

[11]R. Huang, "The inverse problem of bimorph mirror tuning on a beamline," Journal of Synchrotron radiation 18, 930 - 937, (2011).

[12] S. Kokorowski, "Analysis of adaptive optical elements made from piezolectric bimorphs," J. Opt. Soc. Am. 69, 181-187 (1979).

[13]P. Halevi, "Bimorph piezoelectric flexible mirror: graphical solution and comparison with experiment," J. Opt. Soc. Am. 73, 110-113 (1983).

[14] Y. Ning, W. Jiang, N. Ling, and C. Rao, "Response function calculation and sensitivity comparison analysis of various bimorph deformable mirrors," Opt. Express 15, 12030-12038 (2007). 